Article

\title{
Fatigue Analysis of the Oil Offloading Lines in FPSO System under Wave and Current Loads
}

\author{
Xu Zhang ${ }^{1,2}$, Wenchi $\mathrm{Ni}^{1,2, * \mathbb{C}}$ and Liping Sun ${ }^{3}$ \\ 1 School of Marine Engineering and Technology, Sun Yat Sen University, Zhuhai 519082, China; \\ zhangxsysu@gmail.com \\ 2 Southern Marine Science and Engineering Guangdong Laboratory Zhuhai, Zhuhai 519082, China \\ 3 Deepwater Engineering Research Center, Harbin Engineering University, Harbin 150001, China; \\ sunlpheu@gmail.com \\ * Correspondence: niwch@mail.sysu.edu.cn
}

check for updates

Citation: Zhang, X.; Ni, W.; Sun, L. Fatigue Analysis of the Oil Offloading Lines in FPSO System under Wave and Current Loads. J. Mar. Sci. Eng. 2022, 10, 225. https:// doi.org/10.3390/jmse10020225

Academic Editors: Dejan Brkić and José A.F.O. Correia

Received: 24 November 2021

Accepted: 1 February 2022

Published: 8 February 2022

Publisher's Note: MDPI stays neutral with regard to jurisdictional claims in published maps and institutional affiliations.

Copyright: (C) 2022 by the authors. Licensee MDPI, Basel, Switzerland. This article is an open access article distributed under the terms and conditions of the Creative Commons Attribution (CC BY) license (https:// creativecommons.org/licenses/by/ $4.0 /)$.

\begin{abstract}
In this paper, fatigue analysis of oil offloading lines (OOLs) in the floating production storage and offloading (FPSO) catenary anchor leg mooring (CALM) buoy offloading system under wave and current loads in the West Africa Sea area is carried out by the numerical simulation method. The hydrodynamic coupling response is calculated, and fatigue damage is analyzed. Firstly, the numerical model is verified by comparison with the experimental results. Then, according to the environmental statistics in West Africa, the influence of various parameters on the fatigue damage of OOLs is analyzed, including tension characteristics, wave parameters, and structural parameters. Additionally, the effect of current load is studied. Results show that accumulated fatigue damage mainly occurs near the CALM buoy and is mainly caused by the $0^{\circ}$ wind wave. Appropriately reducing the cover length of buoyancy material and increasing the wall thickness can reduce fatigue damage. Moreover, the effect of the shuttle tanker can increase the fatigue damage of the OOL near the CALM buoy by about 1.5 times, and the effect of vortex-induced vibration can increase the fatigue damage of the OOL in the middle part by up to 5-10 times.
\end{abstract}

Keywords: oil offloading line (OOL); wave load; vortex-induced vibration; floating production storage and offloading (FPSO); fatigue; simulations

\section{Introduction}

The sea conditions in West Africa are mild all year round, and the combination of floating production storage and offloading (FPSO) + oil offloading lines (OOLs) + catenary anchor leg mooring (CALM) buoy + shuttle tanker has been widely used for deep-water oil transportation in this sea area [1,2]. In this combination, OOLs are important structures for oil transportation, with a span of more than $2000 \mathrm{~m}$, and their safety is of great concern [3]. This paper will study the fatigue characteristics and parameter sensitivity of the OOL according to the environmental statistics in the West Africa Sea area.

Many studies have been published on the fatigue damage of marine pipelines. Liu et al. [4] studied the coupling effect of direct waves and currents in the context of fatigue damage assessment and proposed a first-order correction factor involving the ratio of current and wave velocities to evaluate the environmental load coupling effect. Arzaghi et al. [5] proposed a probabilistic method to simulate the pitting and corrosion fatigue degradation process of submarine pipelines by using a dynamic Bayesian network to model the time property and growth rate of change. Zhang et al. [6] established a numerical model for the fatigue behavior of multiple coplanar cracks in offshore pipelines under cyclic tensile loading and studied the important parameters that affect fatigue crack growth. Al-Janabi et al. [7] carried out model experiments to study the influence of different parameters on fatigue damage near the touchdown zone of a steel catenary riser at the seabed. 
Although these studies mainly focused on the fundamental fatigue characteristics of materials or numerical models on fatigue behavior, most of them are mechanism studies, so the research objects are mostly simple and typical pipeline structures. However, for practical OOL structures in FPSO offloading systems, related fatigue analysis and design optimization research are insufficient. Chaudhury [8] proposed a "U"-type oil pipeline and made a comparative analysis of the " $\mathrm{U}$ "-type oil pipeline and the " $\mathrm{W}$ "-type oil pipeline, including strength characteristics, fatigue characteristics, economic advantages, etc. Through this research, it was found that the " $\mathrm{U}$ "-type oil pipeline is superior to the " $\mathrm{W}$ "-type oil pipeline in fatigue behavior. Odru et al. [9] analyzed the fatigue characteristics of oil pipelines based on the spectrum analysis method. The results show that the fatigue damage of the pipeline mainly occurs near the buoy. It is difficult for a 22-inch steel pipeline to meet the fatigue requirements, so a buoy submerged in the water is recommended to reduce the wave effect. However, in this analysis, only the first-order wave force is considered. Based on their experience in engineering design in West Africa, Montbarbon et al. [10] proposed that increasing the effective tension of the oil pipeline is conducive to the improvement of its fatigue life. They also proposed that the fatigue characteristics of the oil pipeline should be considered in the case of shuttle tanker operation. Drumond et al. [11] conducted a literature review on failure events experienced by the industry concerning pipelines, risers, and umbilical cables and described their causes, consequences, and severity. He et al. [12] analyzed the effect of pan length on the fatigue life of submarine pipelines, finding that increasing the gap between the suspended pipeline and the seabed is beneficial to enhance the fatigue life of the suspended pipeline.

Due to the fatigue failure of oil pipelines, many accidents such as crude oil leakage have occurred, resulting in serious economic losses and environmental pollution [13,14]. Therefore, it is of great importance to improve the fatigue design knowledge of oil pipelines. However, as can be seen from the above overview, research on the fatigue characteristics of oil offloading lines in the FPSO CALM buoy offloading system is insufficient. Although current studies have paid attention to the effects of pipeline layout and buoy position on fatigue damage, the influence of factors such as motion and tension distribution, wave type, wave direction, coverage length of buoyancy material, outer diameter, wall thickness, shuttle tanker, and current loads on OOL fatigue damage is still not fully studied. Therefore, the purpose of this paper is to comprehensively analyze the influence of the above factors on OOL fatigue damage and provide suggestions for relevant design.

The outline of the rest of this paper is as follows: In Section 2, the methodology for numerical modeling and fatigue calculation is described. In Section 3, the verification of the numerical model is introduced. In Section 4, the calculation results are analyzed, specifically the influence of different parameters on OOL fatigue damage. Section 5 presents the conclusions.

\section{Numerical Model of the Offloading System}

In this paper, a numerical model of the FPSO CALM buoy offloading system is established according to the principal dimensions of the research object in the experimental investigation previously conducted by the authors' team [15]. Firstly, the response amplification operator (RAO), additional mass, and radiation damping of the structures are obtained by the frequency domain analysis using AQWA software (ANSYS, Inc., Canonsburg, PA, USA), and on this basis, the dynamic behavior of the structures are studied using Orcaflex software (Orcina Ltd., Ulverston, Cumbria, UK). Additional damping equal to $5-10 \%$ of the critical damping value is added to each degree of freedom of the structures, the specific value of which is determined by comparison with the corresponding free attenuation experimental results, as well as the RAO experimental results, in typical wave angles. Then, the hydrodynamic responses of the structures are calculated, and the accuracy of the numerical model is verified by comparison with the corresponding experimental results. 


\subsection{Principal Dimensions of the Numerical Model}

The schematic diagram of the numerical model is shown in Figure 1, and the top view of the system is shown in Figure 2. In the ballast condition, the FPSO and shuttle tanker will have a larger motion amplitude under wave load and have a greater impact on the OOL's fatigue damage. Therefore, in the fatigue analysis of this paper, the FPSO and shuttle tanker are both in ballast conditions. For principal dimensions and related parameters of the model, refer to the model experiment previously conducted by the authors' team [15].

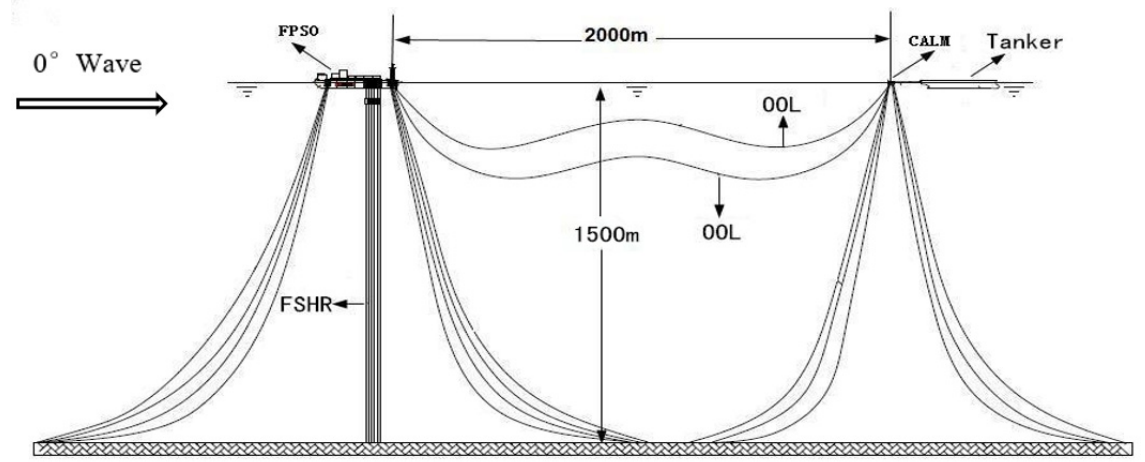

Figure 1. Schematic diagram of the numerical model of the FPSO CALM buoy offloading system.

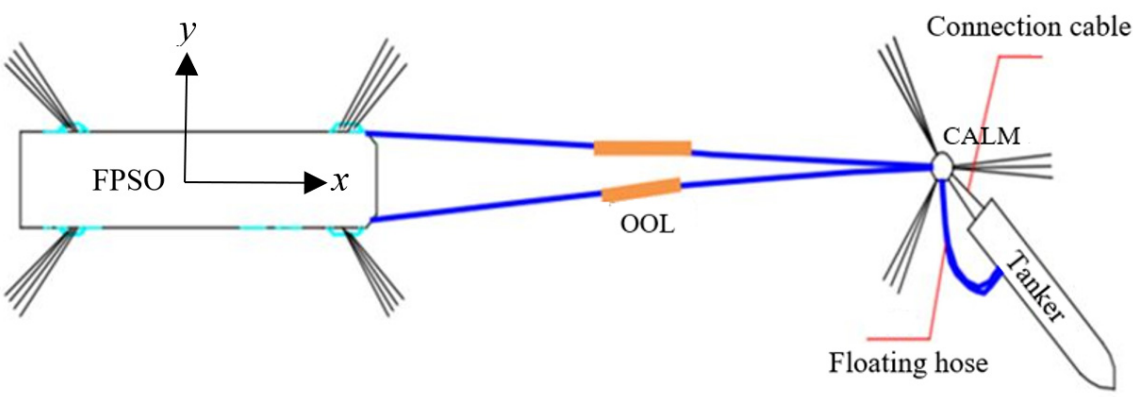

Figure 2. Top view of the FPSO CALM buoy offloading system.

The fatigue analysis in this paper mainly focuses on the OOLs, the specific parameters of which are as follows: The OOL consists of two 20-inch $(508 \mathrm{~mm})$ diameter steel pipes, the material of which is Det Norske Veritas (DNV) E460 steel. Buoyancy materials are assembled in the middle of each pipeline to make it form a " $\mathrm{W}$ " shape after being subjected to buoyancy, as shown in Figure 3.

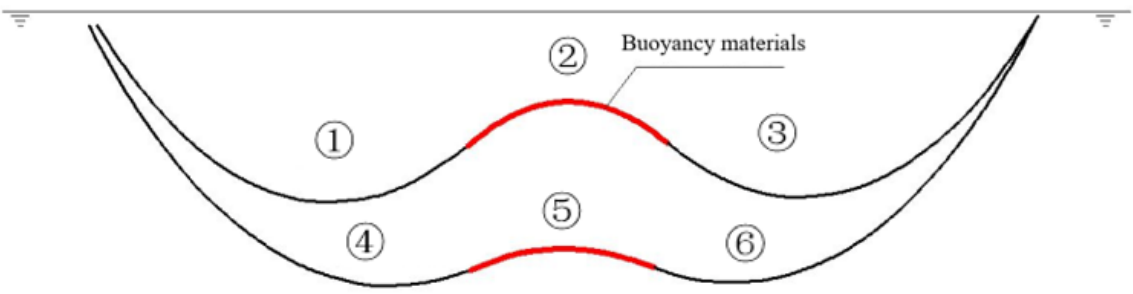

Figure 3. Schematic diagram of the oil offloading lines.

In the spatial arrangement, the two oil pipelines are distributed up and down. The upper OOL is shorter $(2140 \mathrm{~m}$ ) and is connected to the portside of the FPSO and CALM buoy. The lower OOL is longer $(2220 \mathrm{~m})$ and is connected to the starboard side of the FPSO and CALM buoy. The detailed attributes of each segment (as shown in Figure 3) of the OOLs are shown in Table 1. 
Table 1. Detailed attributes of the OOLs.

\begin{tabular}{ccccccc}
\hline Segments & $\begin{array}{c}\text { Outer Diameter } \\
(\mathbf{m})\end{array}$ & $\begin{array}{c}\text { Inner Diameter } \\
(\mathbf{m})\end{array}$ & $\begin{array}{c}\text { Length } \\
(\mathbf{m})\end{array}$ & $\begin{array}{c}\text { Dry Weight } \\
(\mathbf{t} / \mathbf{m})\end{array}$ & $\begin{array}{c}\text { Wet Weight } \\
(\mathbf{t} / \mathbf{m})\end{array}$ & $\begin{array}{c}\text { Tensile Rigidity } \\
\left(\mathbf{k N} / \mathbf{m}^{\mathbf{2}}\right)\end{array}$ \\
\hline (1) and (3) & 0.508 & 0.471 & 860 & 0.2206 & 0.01285 & $5.96 \times 10^{6}$ \\
\hline (2) & 1.000 & 0.471 & 420 & 0.460 & -0.345 & $5.96 \times 10^{6}$ \\
\hline (5) & 1.000 & 0.471 & 360 & 0.460 & -0.345 & $5.96 \times 10^{6}$ \\
\hline (4) and (6) & 0.508 & 0.471 & 930 & 0.2206 & 0.01285 & $5.96 \times 10^{6}$ \\
\hline
\end{tabular}

${ }^{1}$ The buoyancy material is a hollow semi-cylinder with an inner diameter of $508 \mathrm{~mm}$ and an outer diameter of $1000 \mathrm{~mm}$, and its density is $0.4 \mathrm{MT} / \mathrm{m}^{3}$.

The numerical model of the buoy is established by combining the Morison element with the disc element, and the OOLs are connected to the buoy at the top. The numerical model of the buoy and corresponding mooring lines and OOLs is shown in Figure 4, the detail of which has been introduced in the authors' previous work [16]. The other end of the OOLs is connected to the FPSO (see Table 2 for the specific coordinates of the OOL connecting points on the FPSO and buoy).

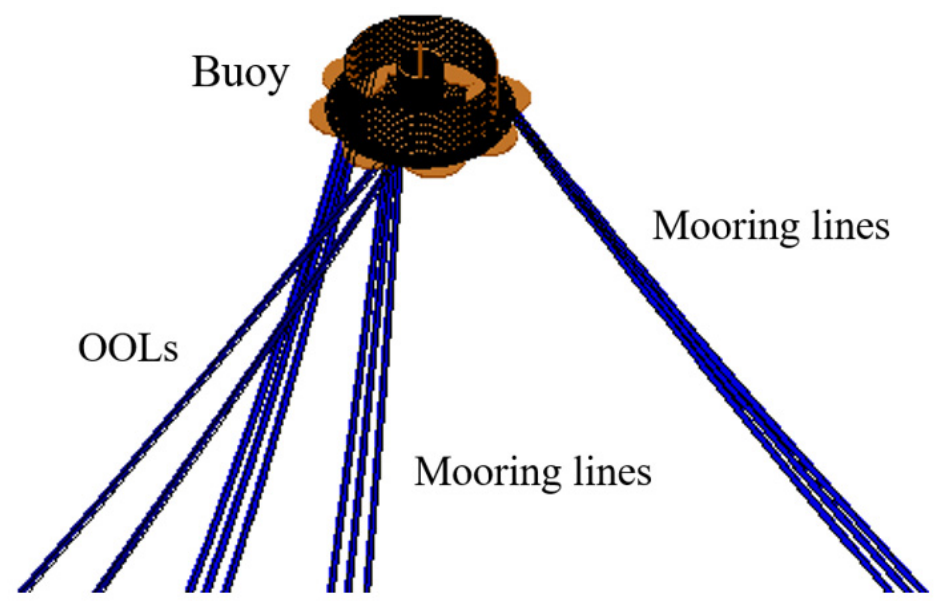

Figure 4. Numerical model of the buoy and the corresponding mooring lines and OOLs.

Table 2. Coordinates of the OOL connecting points on FPSO and buoy.

\begin{tabular}{ccccccc}
\hline \multirow{2}{*}{ OOL } & \multicolumn{3}{c}{ FPSO (Relative to the Center of } & \multicolumn{3}{c}{$\begin{array}{c}\text { Buoy (Relative to the Center of } \\
\text { Underside) }\end{array}$} \\
\cline { 2 - 7 } & $\boldsymbol{X}(\mathbf{m})$ & $\boldsymbol{Y}(\mathbf{m})$ & $\boldsymbol{Z}(\mathbf{m})$ & $\boldsymbol{X}(\mathbf{m})$ & $\boldsymbol{Y ( m )}$ & $Z \mathbf{~ ( m ) ~}$ \\
\hline Port side & 147.5 & 27.1 & 33.3 & -2.118 & 1.4 & 0 \\
\hline Starboard side & 145.4 & -29.1 & 33.3 & -2.118 & -1.4 & 0 \\
\hline
\end{tabular}

\subsection{Model of Loads}

In the numerical model, the FPSO and buoy are rigid bodies, and their motion equations can be derived from Newton's second law. The loads applied to the structures are calculated and taken into the motion equations to solve the six-degrees-of-freedom motion of the FPSO and buoy. Refer to Reference [17] for details. The loads to be calculated mainly include the wave load (both the first-order wave force and the second-order wave force), current load, and the mooring line and OOL's force.

\subsubsection{Mooring Line and OOL's Force}

In this paper, the lumped mass model is adopted for mooring lines and OOLs. The schematic diagram of the line segments is shown in Figure 5. The line element supports 
the flexibility of the line to have axial displacement, torsion, tension, and bending. More details can be found in the authors' previous work [16].

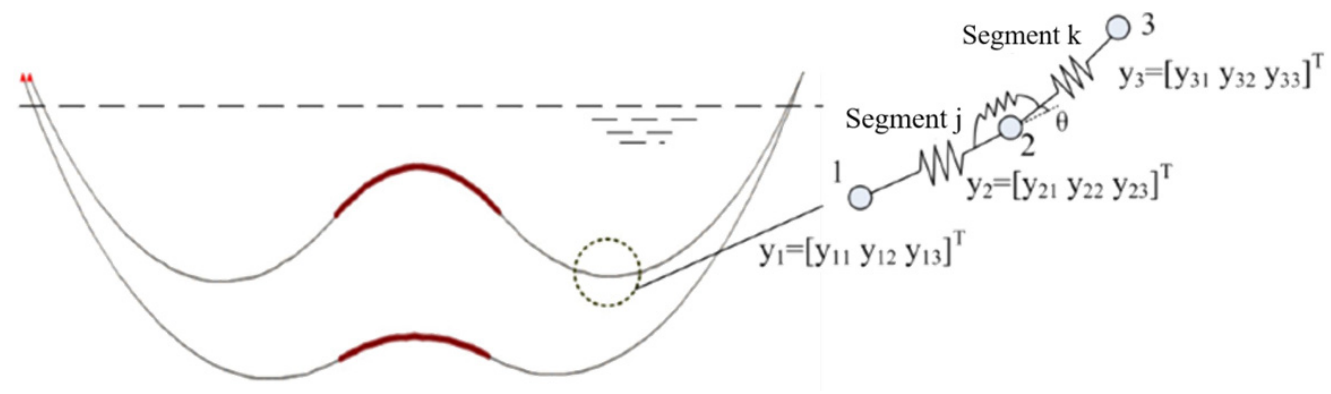

Figure 5. Schematic diagram of line segments in lumped mass model.

\subsubsection{Wave Load}

For the FPSO and shuttle tanker, wave loads are calculated based on potential flow theory [18], and for the CALM buoy and OOLs, wave loads are calculated by slender-body expressions based on Morrison equations.

\subsubsection{Current Load}

The current force and moment can be denoted as follows:

$$
\begin{aligned}
F_{x c} & =\frac{1}{2} C_{x c}\left(\varphi_{c R}\right) \rho_{c} V_{c R}^{2} T L \\
F_{y c} & =\frac{1}{2} C_{y c}\left(\varphi_{c R}\right) \rho_{c} V_{c R}^{2} T L \\
M_{x y c} & =\frac{1}{2} C_{x y c}\left(\varphi_{c R}\right) \rho_{c} V_{c R}^{2} T L^{2}
\end{aligned}
$$

where $F_{x c}, F_{y c}$. and $M_{x y c}$ are longitudinal fluid force, lateral fluid force, and yaw fluid torque. $C_{x c}, C_{y c}$, and $C_{x y c}$ are longitudinal fluid force coefficient, transverse fluid force coefficient, and yaw fluid torque coefficient. $\rho_{c}$ is the density of seawater $\left(\mathrm{kg} / \mathrm{m}^{3}\right), T$ is the average draft, and $V_{c R}$ is the relative velocity. The coefficients can be determined based on Oil Companies International Marine Forum (OCIMF) [19].

\subsection{Method of Fatigue Analysis}

Based on the hydrodynamic coupling calculation results of the system, the S-N curve method, which is widely adopted at present [20,21], is used to analyze the fatigue characteristics of the OOLs. According to the requirements of DNV-RP-C203 specification [22], the DNV-E curve is used for fatigue calculation in this paper. The parameters of the S-N curve used are shown in Table 3. Based on the above method, the fatigue damage of the OOL is calculated by Orcaflex software. The S-N curve method used in this paper is described as follows:

Table 3. Details of the DNV-E curve.

\begin{tabular}{ccccccc}
\hline \multirow{2}{*}{ Type of S-N Curve } & \multirow{2}{*}{ SCF } & \multicolumn{3}{c}{$\mathbf{N}<\mathbf{1 0 6}$} & \multicolumn{2}{c}{$\mathbf{N}>\mathbf{1 0 6}$} \\
\cline { 3 - 6 } & & $\mathbf{A}$ & $\mathbf{m}$ & $\mathbf{A}$ & $\mathbf{m}$ \\
\hline DNV-E & 1.13 & $4.0738 \times 10^{11}$ & 3 & $2.2387 \times 10^{15}$ & 5 \\
\hline
\end{tabular}

\subsubsection{Calculation of Time-Domain Fatigue Stress}

According to the established hydrodynamic numerical simulation model of the FPSO oil transportation system, the time-domain curve of fatigue stress of the OOL is calculated. 
Assuming that the oil pipeline is isotropic, the fatigue stress can be obtained by the following formula:

$$
\sigma=\sigma_{A}+\sigma_{M}=\frac{T}{A}+C r\left(M_{x} \sin \theta-M_{y} \cos \theta\right) / I_{x y}
$$

where $A$ is the effective area of the section, $I_{x y}$ is the second-order stress moment, $\sigma_{A}$ is the axial stress, $\sigma_{M}$ is the bending stress, $T$ is axial tension, $\mathrm{Cr}$ is the bending moment load factor, and $M_{x}$ and $M_{y}$ are the bending moments.

\subsubsection{Rain-Flow Counting Method}

After obtaining the time-domain calculation results of OOL fatigue stress in the irregular wave, the average stress, stress amplitude, and stress cycle times of each node of the oil pipeline are obtained by the rain-flow counting method.

\subsubsection{Stress Correction}

The stress obtained by the rain-flow counting method is corrected as follows:

$$
\begin{array}{cc}
S_{e}=S_{r} /\left(1-S_{m} / S M T S\right) & 0<S_{m}<S M T S \\
S_{e}=S_{r} & -S M T S<S_{m}<0
\end{array}
$$

where $S_{r}$ is the actual stress, $S_{m}$ is the average stress, and SMTS is the ultimate tensile strength of the material.

The stress concentration factor and wall thickness correction factor are used to consider the influence of welding. The specific formula is:

$$
S=S_{e} \cdot S C F \cdot\left(\frac{t_{f a t}}{t_{r e f}}\right)^{k}
$$

where $S_{e}$ is the nominal stress amplitude, SCF is the stress concentration factor, and $\left(t_{f a t} / t_{r e f}\right)^{k}$ is the thickness correction factor, which is applicable to the case that the actual wall thickness $t_{f a t}$ exceeds the reference thickness $t_{r e f}=25 \mathrm{~mm}$.

\subsubsection{Calculation of Fatigue Damage by S-N Curve}

The fatigue damage is calculated according to the following formula:

$$
\log N=\log a-m \log (S)
$$

where $N$ is the number of stress cycles, $S$ is the stress amplitude, and $a$ and $m$ are material coefficients.

\subsubsection{Calculation of Accumulated Fatigue Damage}

In this paper, the alternating load of variable amplitude needs to be considered in the fatigue analysis. Therefore, Miner's linear accumulation criterion is used to calculate the fatigue damage accumulation results under variable amplitude stress cycle. The expression of Miner's linear accumulation criterion is as follows:

$$
D_{A}=\sum_{i} \frac{n\left(S_{i}\right)}{N\left(S_{i}\right)}
$$

where $n\left(S_{i}\right)$ is the actual number of stress cycles with amplitude $S_{i}$, and $N\left(S_{i}\right)$ is the total number of stress cycles in the case of fatigue failure of the structure under this stress cycle.

\subsection{Environment Condition}

The working location of the oil offloading system studied in this paper is the sea area of West Africa. In this sea area, the wave condition is relatively mild, the main 
wave component is the swell with a long period, and there are wind waves in some parts. Specifically, the wave components in West Africa can be divided into main swell, secondary swell, and wind wave. The Joint North Sea Wave Atmosphere Program (JONSWAP) spectrum [23] is used to simulate the individual action of swell, and the Ochi Hubble spectrum [24] is used to simulate the joint action of wind wave and swell. For the single wind wave condition, the wind wave component in the Ochi Hubble spectrum is used for the numerical simulation. The wave angle is considered as a fixed value in this paper.

The working area of the offloading system analyzed in this paper is a certain sea area with a depth of $1500 \mathrm{~m}$ in West Africa, and the considered wave distribution is identified according to the observation statistics of the joint wave distribution in West Africa. Three wave conditions need to be considered in this paper: main swell, secondary swell, and wind wave. Their significant wave height $\left(H_{s}\right)$-average period $\left(T_{p}\right)$ distribution characteristics are shown in Tables 4-6.

Table 4. Wave height-period distribution of the main swell in West Africa.

\begin{tabular}{ccccccccccc}
\hline $\boldsymbol{H}_{\boldsymbol{s}}(\mathbf{m})$ & \multicolumn{9}{c}{ Exposure Time (hour) } \\
\hline 0.625 & 713.1 & 832.2 & 843.6 & 632.5 & 249.7 & 155 & 113.9 & 30.7 & 30.7 \\
\hline 0.875 & 290.8 & 516 & 641.2 & 924.2 & 418.7 & 144.5 & 80.6 & 66.6 & 30.7 \\
\hline 1.375 & 41.2 & 116.5 & 213.7 & 402.1 & 391.6 & 305.7 & 155.9 & 70.1 & 42 \\
\hline 2 & 0 & 0 & 2.6 & 16.6 & 47.3 & 80.6 & 121.8 & 28.9 & 7.9 \\
\hline$T_{p}(\mathrm{~s})$ & 10.5 & 11.5 & 12.5 & 13.5 & 14.5 & 15.5 & 16.5 & 17.5 & 18.5 \\
\hline
\end{tabular}

Table 5. Wave height-period distribution of the secondary swell in West Africa.

\begin{tabular}{ccccccccccccccc}
\hline $\boldsymbol{H}_{\boldsymbol{s}}(\mathbf{m})$ & & \multicolumn{10}{c}{ Exposure Time (hour) } \\
\hline 0.375 & 290.8 & 918.9 & 810.3 & 562.4 & 392.4 & 296.1 & 489.7 & 93.3 & 453.8 & 399.5 & 127 & 109.5 & 42 & 8.8 \\
\hline 0.625 & 139.3 & 477.4 & 501.9 & 404.7 & 381.1 & 229.5 & 266.3 & 199.7 & 114.8 & 108.6 & 96.4 & 72.7 & 18.4 & 0 \\
\hline 0.875 & 6.1 & 6.1 & 78.8 & 54.3 & 66.6 & 72.7 & 12.3 & 24.5 & 42 & 24.5 & 35.9 & 12.3 & 0 & 0 \\
\hline 1.25 & 0 & 0 & 0 & 0 & 6.1 & 0 & 0 & 0 & 6.1 & 6.1 & 0 & 0 & 0 & 0 \\
\hline$T_{p}(\mathrm{~s})$ & 7.5 & 8.5 & 9.5 & 10.5 & 11.5 & 12.5 & 13.5 & 14.5 & 15.5 & 16.5 & 17.5 & 18.5 & 19.5 & 20.5 \\
\hline
\end{tabular}

Table 6. Wave height-period distribution of the wind wave in West Africa.

\begin{tabular}{cccccc}
\hline $\boldsymbol{H}_{\boldsymbol{s}}(\mathbf{m})$ & \multicolumn{5}{c}{ Exposure Time (hour) } \\
\hline 0.375 & 28 & 318 & 1064.3 & 862 & 283.8 \\
\hline 0.85 & 137.5 & 823.4 & 2586.8 & 1081 & 311.9 \\
\hline 1.5 & 0 & 42 & 679.8 & 402.1 & 34.2 \\
\hline 1.625 & 0 & 0 & 33.3 & 35.9 & 16.6 \\
\hline 2 & 0 & 0 & 0 & 16.6 & 2.6 \\
\hline$T_{p}(\mathrm{~s})$ & 3.5 & 4.5 & 6.5 & 6.5 & 7.5 \\
\hline
\end{tabular}

\section{Verification of Numerical Model}

In order to verify the accuracy of the numerical model, the results of the numerical simulation are compared with the results of the previous experimental investigation conducted by the authors' team [15]. Numerical simulation results of CALM buoy motion and portside OOL top tension are compared with the experimental results, including the time-domain curves and frequency domain analysis. The environment condition is the combined action of swell and wind wave, where $H_{s}($ swell $)=2.6 \mathrm{~m}, T_{p}($ swell $)=15.9 \mathrm{~s}$; $H_{s}($ wind wave $)=2.05 \mathrm{~m}$, and $T_{p}$ (wind wave) $=6.7 \mathrm{~s}$. The wave direction is $0^{\circ}$ (as shown in Figure 1). 
The numerical and experimental results of surge, heave, and pitch motions of the CALM buoy, as well as the top tension (in the experiment, a force sensor is set at the connection between OOL and buoy, and the force measured by the force sensor is the top tension), of the portside OOL are shown in Figures 6-9, respectively. As shown in the figures, the numerical simulation results of the CALM buoy's motion and OOL's top tension are generally in good agreement with the experimental values, except that the numerical results of pitch motion amplitude of the CALM buoy in Figure 8 are slightly larger than the experimental results. This should be due to the ignorance of a part of the fluid viscous damping in the hydrodynamic calculation method based on the potential flow theory used in this paper.

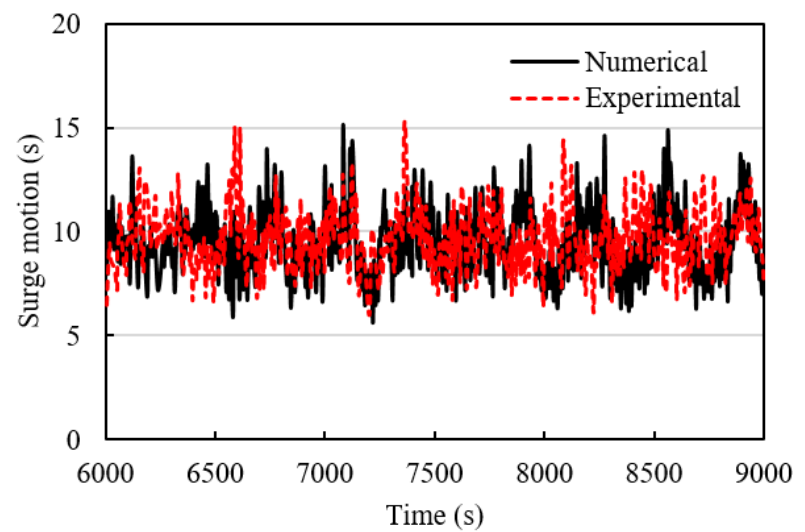

(a) Time-domain response diagram

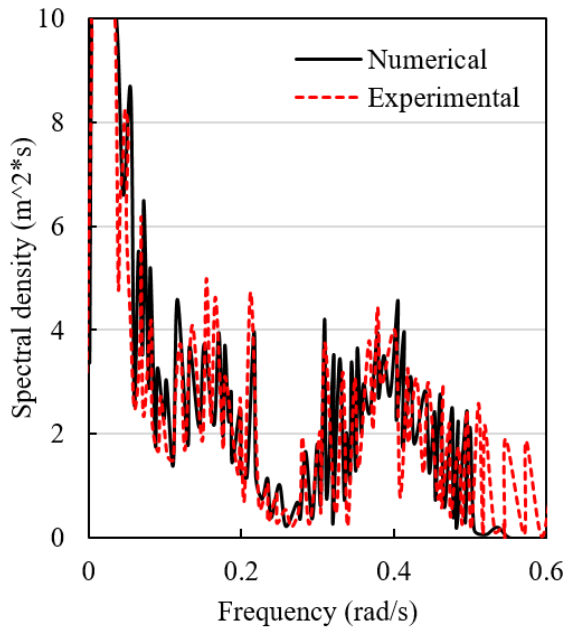

(b) Frequency response diagram

Figure 6. Surge motion of the CALM buoy.

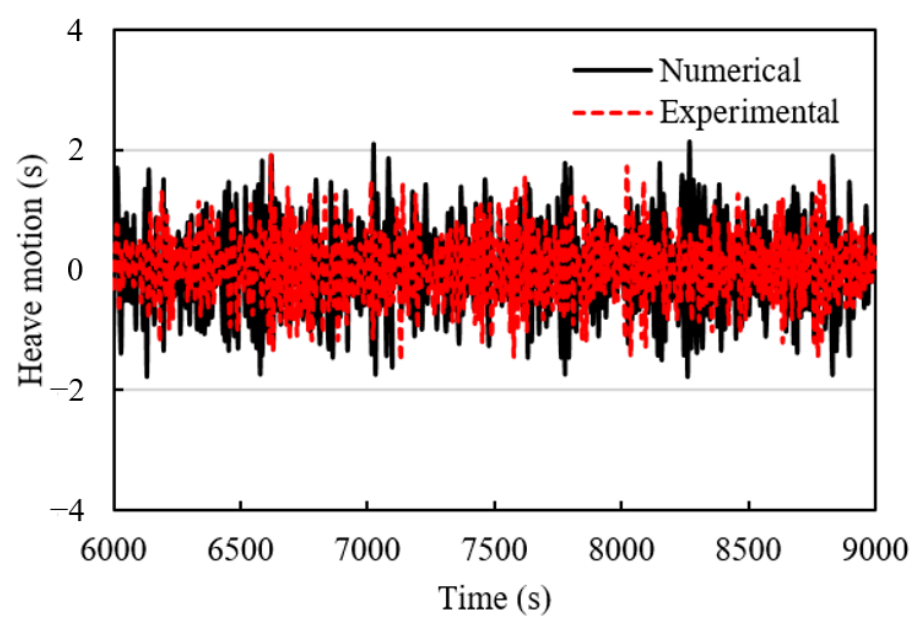

(a) Time-domain response diagram

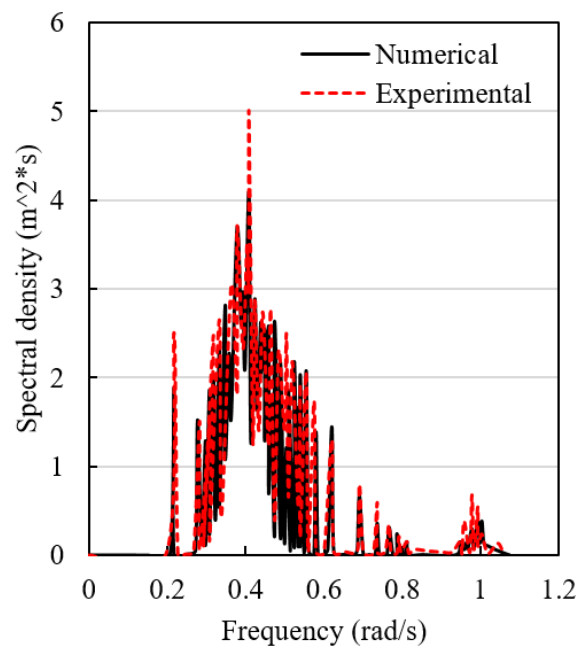

(b) Frequency response diagram

Figure 7. Heave motion of the CALM buoy.

Considering that the stress of the OOL is the most important factor affecting its fatigue damage, the numerical simulation results of the $\mathrm{OOL}_{\mathrm{p}}$ top tension are compared with the experimental results in detail, as shown in Table 7. It can be seen that the numerical simulation errors of maximum value, average value, and standard deviation are very small, all less than $5.7 \%$. Only the error of the minimum value is relatively large, reaching $17.1 \%$. Considering that the error of the minimum value is not that relevant because, for design 
purposes, the minimum top tension is not that important, the overall calculation error is within the acceptable range, proving that the numerical simulation model is reliable.

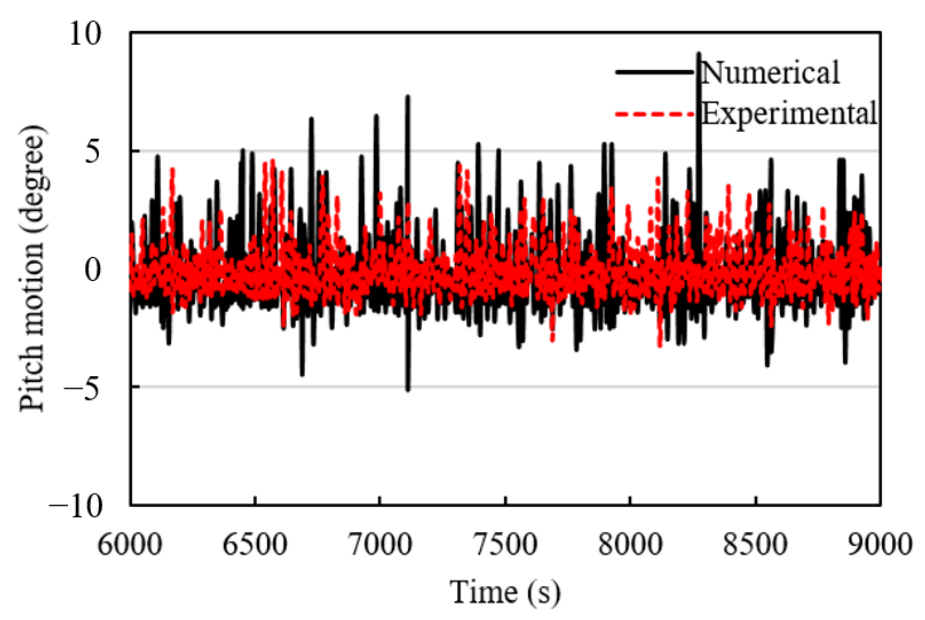

(a) Time-domain response diagram

Figure 8. Pitch motion of the CALM buoy.

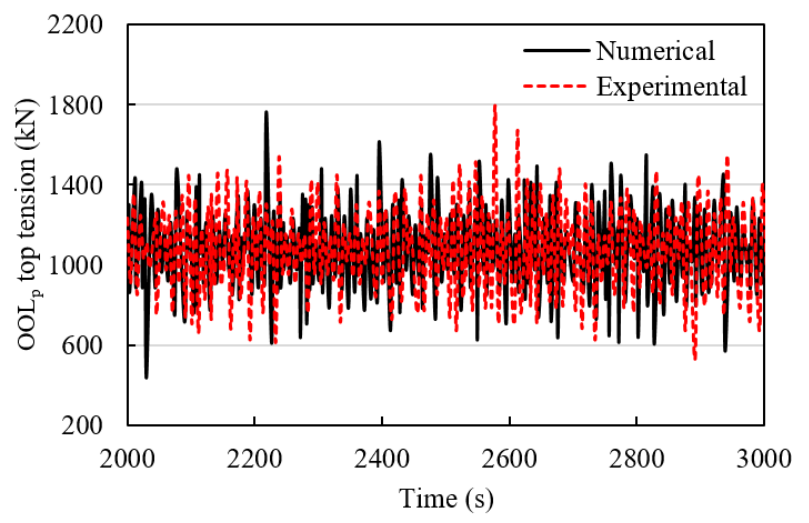

(a) Time-domain response diagram

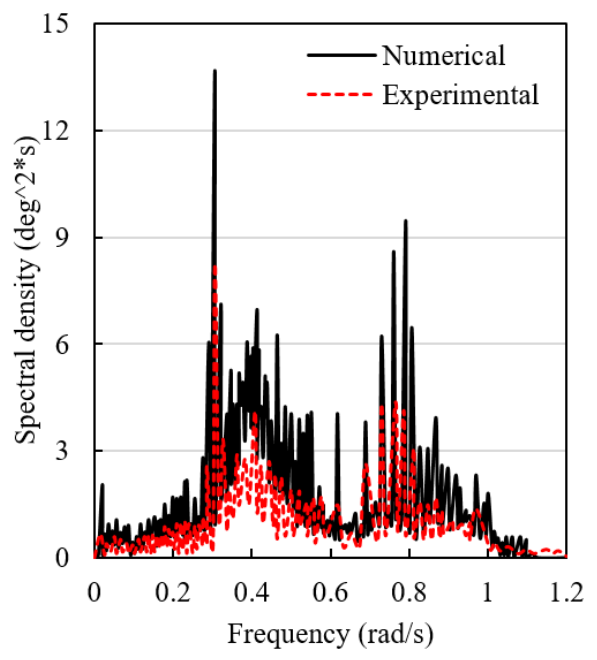

(b) Frequency response diagram

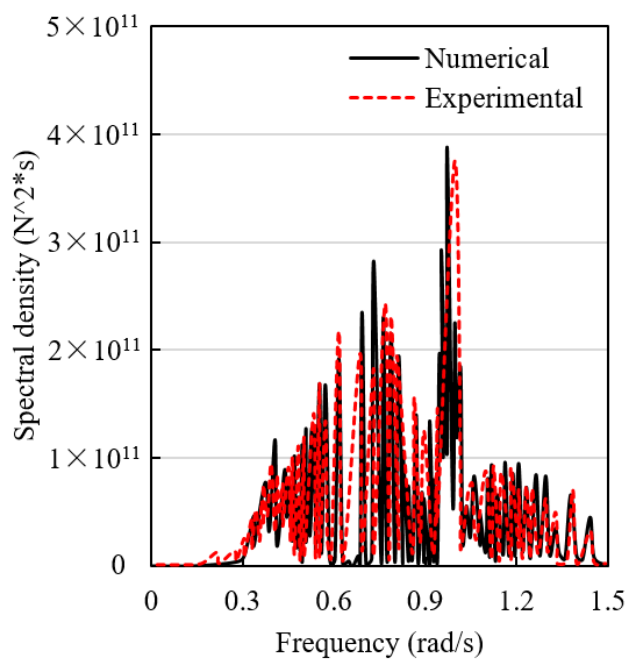

(b) Frequency response diagram

Figure 9. Top tension of the portside OOL.

Table 7. Comparison of experimental and numerical simulation results of $\mathrm{OOL}_{\mathrm{p}}$ top tension.

\begin{tabular}{cccc}
\hline & \multicolumn{3}{c}{ Top Tension of the Portside OOL } \\
\cline { 2 - 4 } & $\begin{array}{c}\text { Numeric Results } \\
\mathbf{( k N )}\end{array}$ & $\begin{array}{c}\text { Experimental Result } \\
\mathbf{( k N )}\end{array}$ & Error (\%) \\
\hline Maximum value & 1762 & 1796 & 1.8 \\
\hline Minimum value & 435 & 525 & 17.1 \\
\hline Average value & 1077 & 1077 & 0 \\
\hline Standard deviation & 250 & 265 & 5.7 \\
\hline
\end{tabular}




\section{Fatigue Analysis}

In this section, the hydrodynamic and fatigue characteristics of the portside OOL are analyzed first, and then the influence of other parameters on the fatigue damage of the OOL is analyzed.

\subsection{Hydrodynamic and Fatigue Characteristics of the OOL}

In order to study the relationship between the dynamic responses of motion and tension of the OOL and its fatigue damage, the coupling analysis of the system in typical working conditions is carried out. The wave condition is the wind wave, $H_{s}=1.5 \mathrm{~m}$, $T_{p}=5.5 \mathrm{~s}$, the wave direction is $0^{\circ}$, and the calculation time is $2000 \mathrm{~h}$.

Figure 10 shows the distribution of maximum motion amplitude of the portside OOL and the fatigue damage, where the abscissa represents the OOL's segment at the corresponding coordinate position (from FPSO to CALM). As shown in Figure 10, along the length of the OOL, there are two peaks of fatigue damage. The first peak is located in the middle segments of the OOL, corresponding to the segment covered by buoyancy material (as shown in the red section in Figure 3). The second peak is near the CALM buoy, where the fatigue damage is much greater than the rest of the OOL. Moreover, there is no obvious correlation between the distribution of motion amplitude and the distribution of fatigue damage. However, in some locations, such as segments covered with buoyancy material or near the CALM buoy, the motion amplitude is smaller due to boundary conditions, but the fatigue damage is greater than the nearby areas.

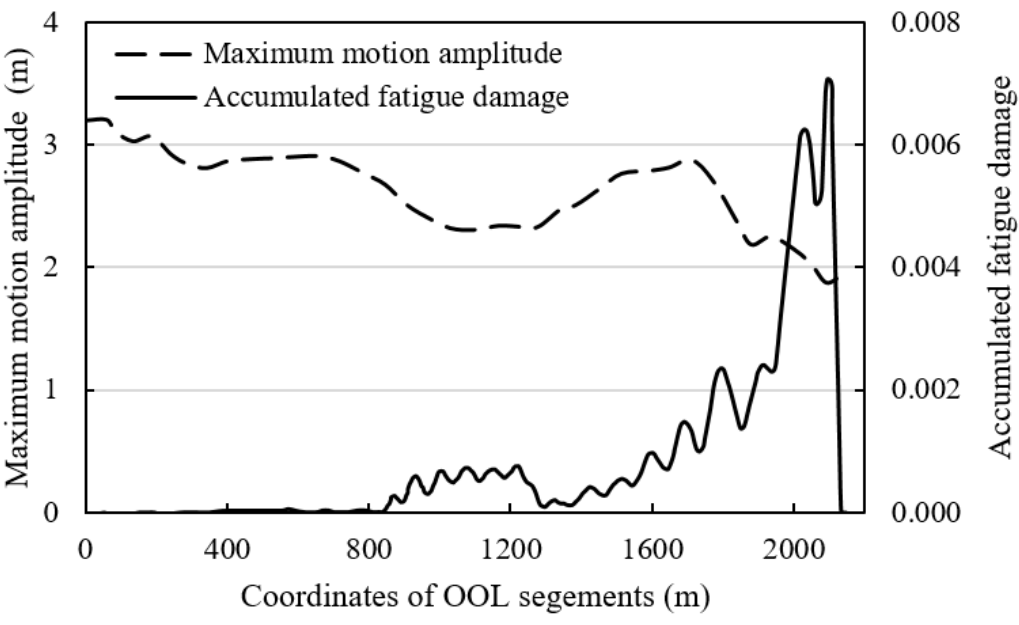

Figure 10. Motion amplitude and fatigue damage distribution along the length of OOL.

Figure 11 shows the distribution of tension (variation amplitude and the maximum value) and fatigue damage along the length of the OOL. As can be seen from the figure, the distribution of fatigue damage shows a clear positive correlation with the distribution of tension variation amplitude, but there is no obvious correlation with the distribution of maximum tension, indicating that the tension variation amplitude is a major factor for fatigue damage. Moreover, the growth rate of fatigue damage is obviously greater for segments covered with buoyancy material or located near the CALM buoy. As shown in Figure 10, both of these two parts of the OOL are subjected to constrained boundary conditions, resulting in a small motion amplitude. This indicates that under similar external force conditions, the constrained boundary conditions may increase the fatigue damage. It should be noted that the maximum stress of the OOL in this case is about $150 \mathrm{MPa}$, which is far lower than the material's yield limit of $460 \mathrm{MPa}$. 


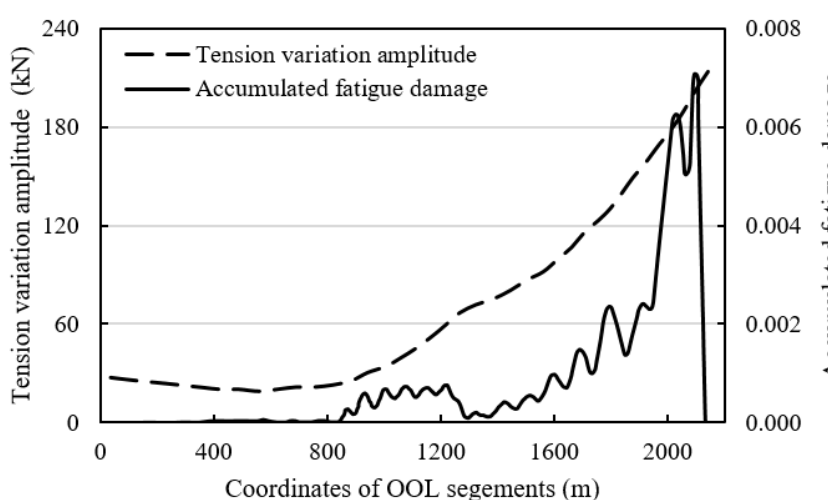

(a) Tension variation amplitude

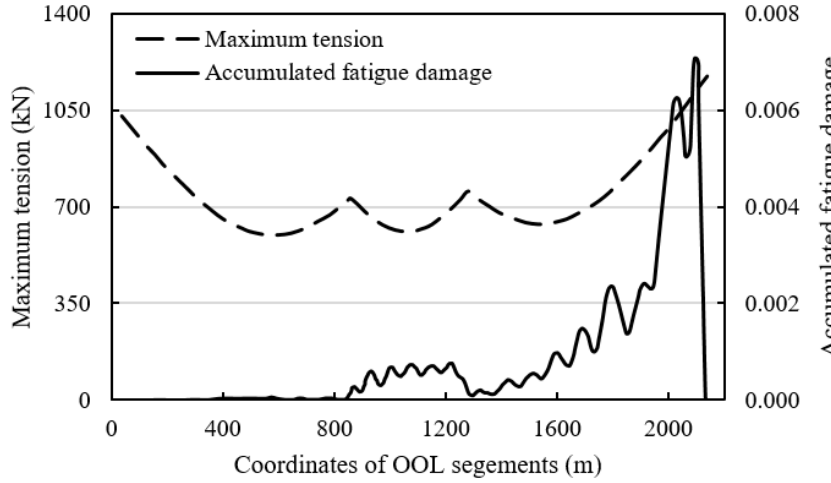

(b) Maximum tension

Figure 11. Tension and fatigue damage distribution along the length of OOL.

\subsection{Influence of Wave Parameters on Fatigue Damage}

In this part, the influences of the type and direction of wave loads on the fatigue damage of the OOL is analyzed.

\subsubsection{Influence of Wave Types}

As mentioned above, the wave components in West Africa can be divided into main swell, secondary swell, and wind wave. In order to analyze the different effects of these three wave conditions on the fatigue damage of the OOL, the analysis of parameter sensitivity of different kinds of wave loads on fatigue damage is carried out. It should be noted that the calculation process is to conduct dynamic analysis under each wave condition based on the wave distribution shown in Tables 4-6. Then, according to the specific exposure time of each wave condition, the fatigue damage of the OOL under each wave condition is accumulated in proportion.

The fatigue damage of the OOL under three different wave conditions is shown in Figure 12. It can be found that fatigue damage mainly occurs in the middle parts of the OOL (covered by buoyancy material) and the parts near the CALM buoy. In addition, the fatigue damage of the former is mainly caused by the swell, and the fatigue damage of the latter is mainly caused by the wind wave. Additionally, the fatigue damage caused by the secondary swell is very small.

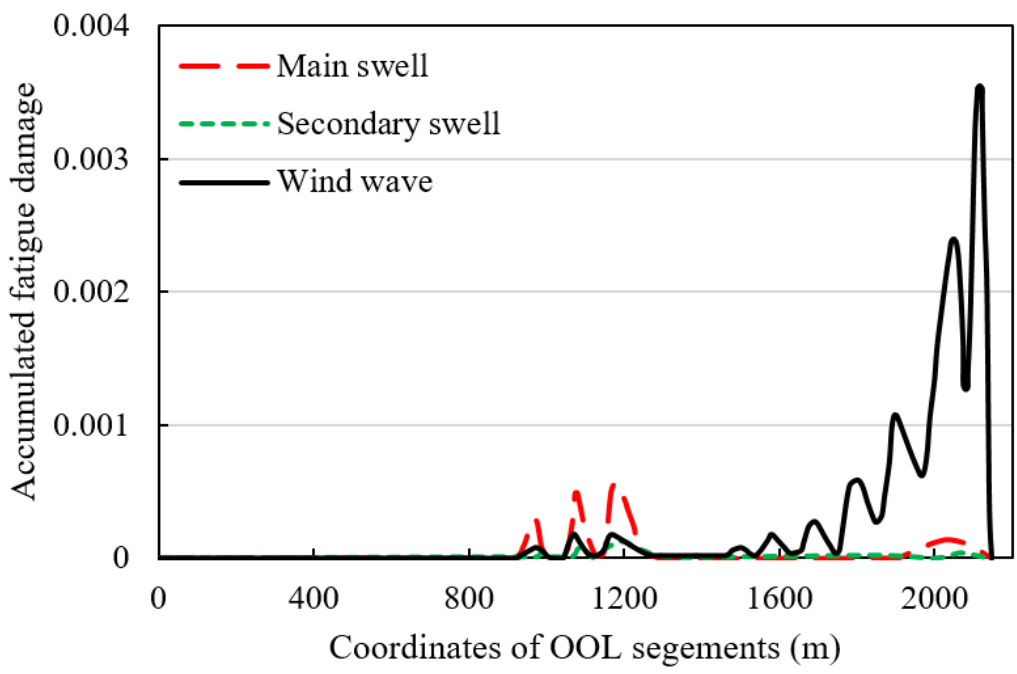

Figure 12. Fatigue damage of OOL under different wave conditions. 


\subsubsection{Influence of Wave Direction}

In order to study the influence of wave direction on the fatigue damage of the OOL, the fatigue damage caused by wave load in different directions in the same period is calculated as shown in Figure 13, where the type of wave load applied is the wind wave, and the calculation method is similar to that in Section 4.2.1. As shown in Figure 13, with the increase in wave direction from $0^{\circ}$ to $90^{\circ}$, the fatigue damage of the OOL decreases obviously. The fatigue damage at $0^{\circ}$ is about 10 times that at $90^{\circ}$, indicating that the surge motion of the CALM buoy caused by the $0^{\circ}$ wave loads is the main cause of the fatigue damage of the OOL.

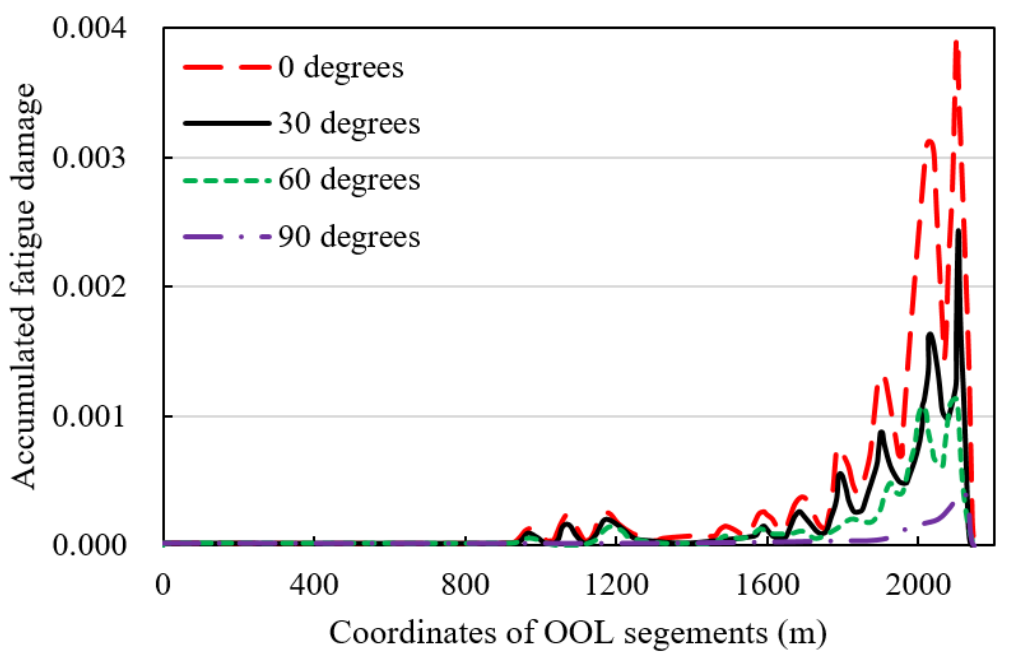

Figure 13. Fatigue damage of OOL under different wave directions.

\subsection{Influence of Structural Parameters of the OOL on Fatigue Damage}

The outer diameter, wall thickness, and coverage area of buoyancy material are important structural parameters of the OOL. This section will analyze the influence of these three parameters on fatigue damage.

\subsubsection{Influence of Coverage Length of Buoyancy Material}

In order to study the influence of coverage length $(L)$ of buoyancy material on the fatigue damage of the OOL, three working conditions ( $L=380 \mathrm{~m}, L=420 \mathrm{~m}$, and $L=460 \mathrm{~m}$ ) are selected for coupling calculation and fatigue damage analysis. The fatigue damage calculation results are shown in Figure 14, and the corresponding spatial distribution shapes of the OOL with different coverage lengths of buoyancy material are shown in Figure 15. The wave load used in this calculation is the $0^{\circ}$ wind wave, with a significant wave height of $1.5 \mathrm{~m}$ and a period of $5.5 \mathrm{~s}$. The calculation time is $2000 \mathrm{~h}$.

Combined with Figures 14 and 15, it can be found that the coverage length of buoyant material can change the spatial distribution shape of the OOL and then change the magnitude and distribution of tension. With the increase in the coverage length of buoyant material, the tension of the OOL will decrease, and the curvature of the overall structure will increase, then the fatigue damage of the structure will increase. Moreover, the variation in fatigue damage is mainly concentrated in the parts near the CALM buoy. Therefore, in order to reduce the fatigue damage caused by wave load, it is suggested that on the premise of meeting the structural strength requirements, the coverage length of buoyancy material can be reduced appropriately. 


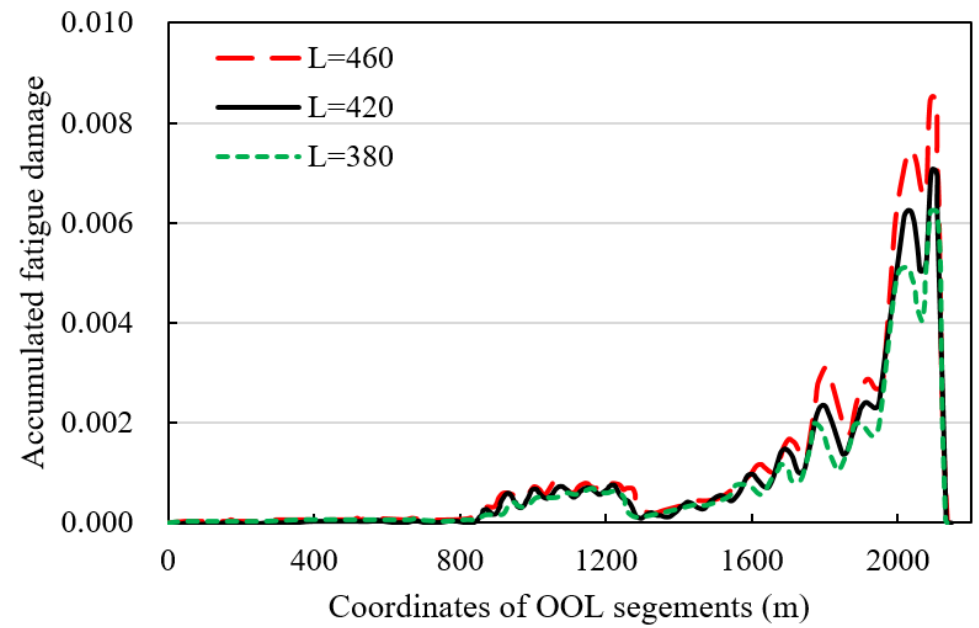

Figure 14. Fatigue damage of OOL with different coverage length of buoyancy material.

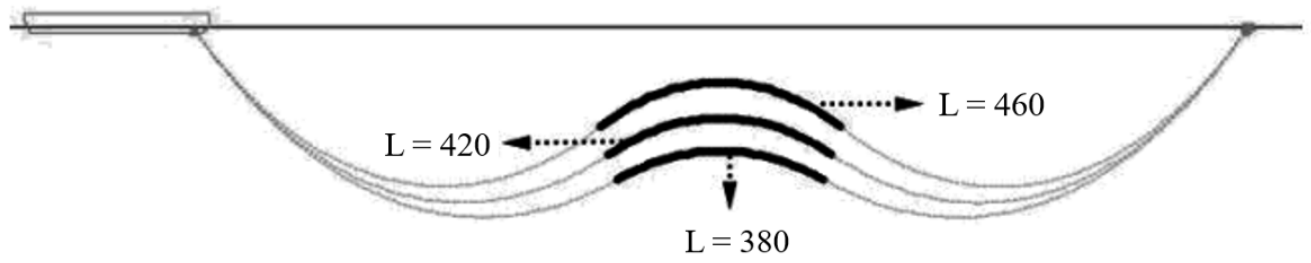

Figure 15. Shapes of OOL with different coverage length of buoyancy material.

\subsubsection{Influence of Outer Diameter}

In order to study the influence of the outer diameter of the OOL on the fatigue damage, keep the wall thickness unchanged in the calculation, and take the outer diameter of $D=513 \mathrm{~mm}, D=508 \mathrm{~mm}$, and $D=503 \mathrm{~mm}$, respectively, for coupling calculation and fatigue analysis. The results are shown in Figure 16. The wave parameters used in the calculation are the same as those in Section 4.3.1.

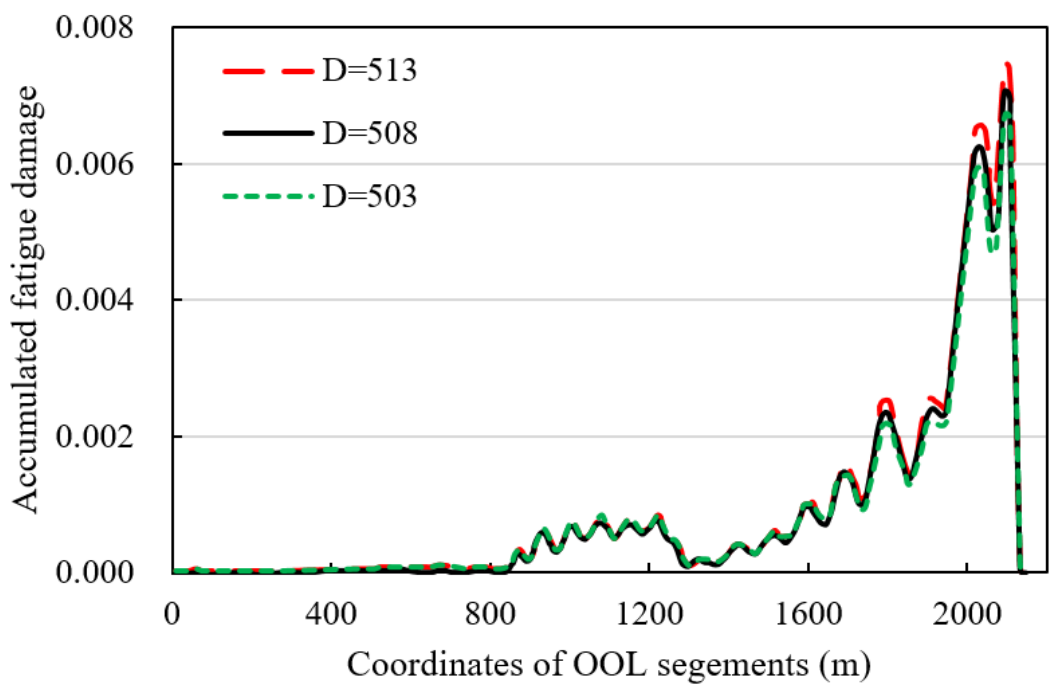

Figure 16. Fatigue damage of OOL with different outer diameters.

As shown in Figure 16, with the increase in the outer diameter of the OOL (the wall thickness remains unchanged), the fatigue damage of the pipeline shows an increasing trend under the same wave conditions. The variation in fatigue damage caused by the change in outer diameter is mainly concentrated in the area near the CALM buoy, however, it is not very obvious. 


\subsubsection{Influence of Wall Thickness}

In this part, the effect of wall thickness (WT) on fatigue damage is studied. Keep the outer diameter unchanged in the calculation, and take the wall thickness of WT $=17 \mathrm{~mm}$, $W T=18 \mathrm{~mm}$, and $W T=21 \mathrm{~mm}$, respectively, for coupling calculation and fatigue analysis. The results are shown in Figure 17.

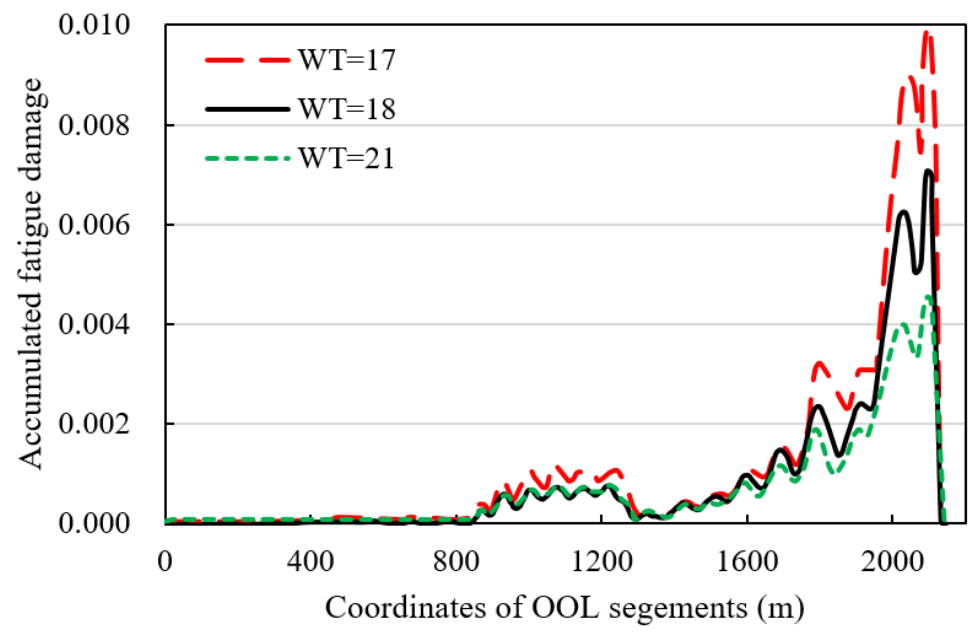

Figure 17. Fatigue damage of OOL with different wall thickness.

As shown in Figure 17, with the increase in the wall thickness of the OOL (the outer diameter remains unchanged), the fatigue damage of the pipeline will be reduced, and the variation in fatigue damage caused by the change in wall thickness is mainly concentrated in the area near the CALM buoy. In addition, compared with the effect of outer diameter (as shown in Figure 16), the effect of wall thickness on fatigue damage is much more significant.

Considering that the increase in wall thickness will also increase the weight of the OOL and then change its spatial distribution shape, now, the influence of wall thickness (as shown in Figure 17) and the influence of outer diameter (as shown in Figure 16) are compared. When the wall thickness increases from $17 \mathrm{~mm}$ to $18 \mathrm{~mm}$, the total weight increases about $5 \%$, and the fatigue damage can be reduced by up to $40 \%$. When the outer diameter increases from $503 \mathrm{~mm}$ to $513 \mathrm{~mm}$, the total weight increases about $2 \%$, and the fatigue damage of the OOL shows an increasing trend rather than decreasing. It can be inferred that for the FPSO OOL, the influence of wall thickness itself on fatigue damage is much more significant than that of weight and spatial distribution shape variation in the OOL caused by the change in wall thickness. Therefore, in order to reduce the fatigue damage caused by the wave load, it is feasible to increase the wall thickness properly.

\subsection{Influence of the Shuttle Tanker}

During the service period of the offloading system, about $20-40 \%$ of the time, it is moored by the shuttle tanker for oil transportation [25]. In order to study the effect of the shuttle tanker on fatigue damage of the OOL, coupling calculation and fatigue analysis of two conditions (with shuttle tanker and without shuttle tanker) are carried out, and the results are shown in Figure 18. The schematic of the shuttle can be seen in Figure 2, and the wave parameters used in the calculation are the same as those in Section 4.3.1. 


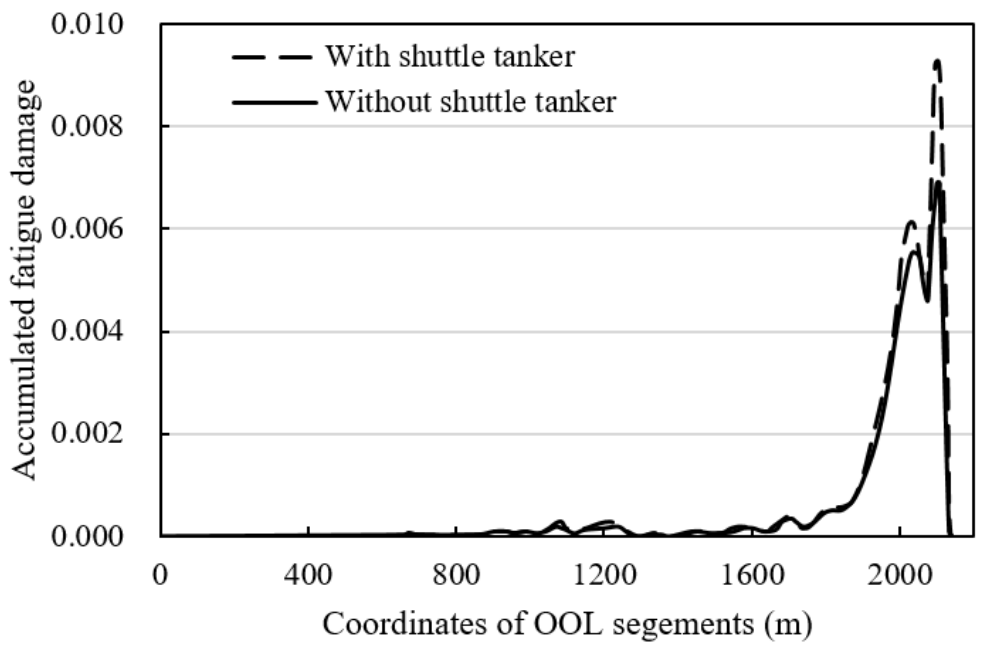

Figure 18. Influence of shuttle tanker on fatigue damage of OOL.

As shown in Figure 18, although shuttle tankers are not directly in contact with the OOL, by influencing the motion state of the CALM buoy, the fatigue damage of the OOL will increase to about 1.5 times. The object of influence is mainly the part of the OOL near the CALM buoy, and the influence on other parts can be basically ignored.

\subsection{Influence of the Flow-Induced Vibration}

Vortex-induced vibration is also one of the important factors leading to fatigue damage of offshore pipelines [26]. In this part, the effect of vortex-induced vibration on the fatigue damage of the OOL will be studied. In this case, the current load is applied. The flow velocity is in the form of shear flow, and the parameters are defined according to the frequently occurring sea conditions in West Africa, as shown in Figure 19. The vortex force is calculated by the vortex tracking model based on the software Orcaflex, in which the value of the lift coefficient $(\mathrm{Cl})$ adopts the table of the lift coefficient compiled by Norberg [27].

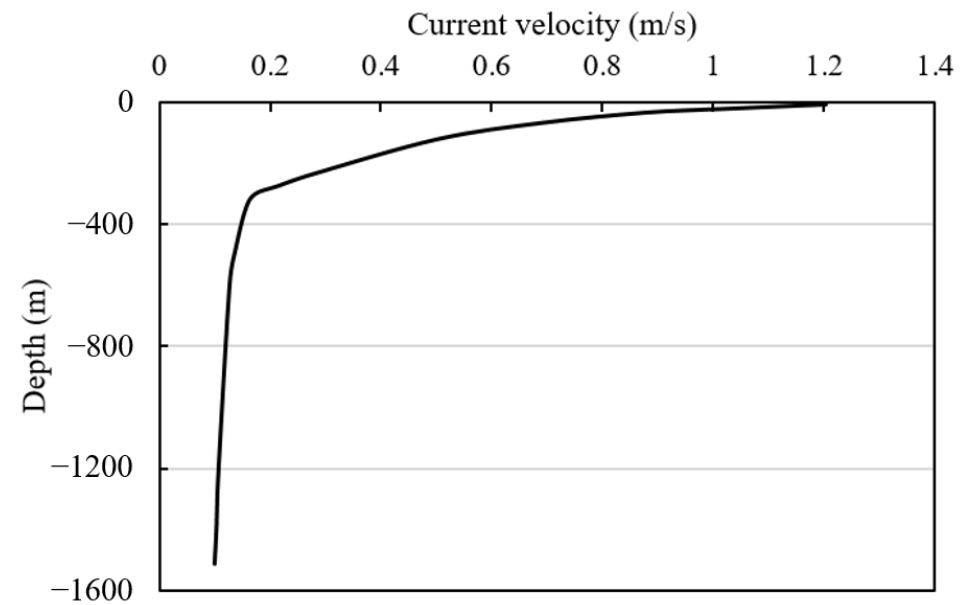

Figure 19. Velocity distribution defined in numerical simulation.

In order to study the effect of vortex-induced vibration on fatigue damage, the fatigue damage of the OOL considering vortex-induced vibration $(\mathrm{Cl}=\mathrm{Cl}(\mathrm{Re}))$ and not considering vortex-induced vibration $(\mathrm{Cl}=0)$ are calculated. The fatigue damage distribution of the OOL when the current direction is $45^{\circ}$ is shown in Figure 20. 


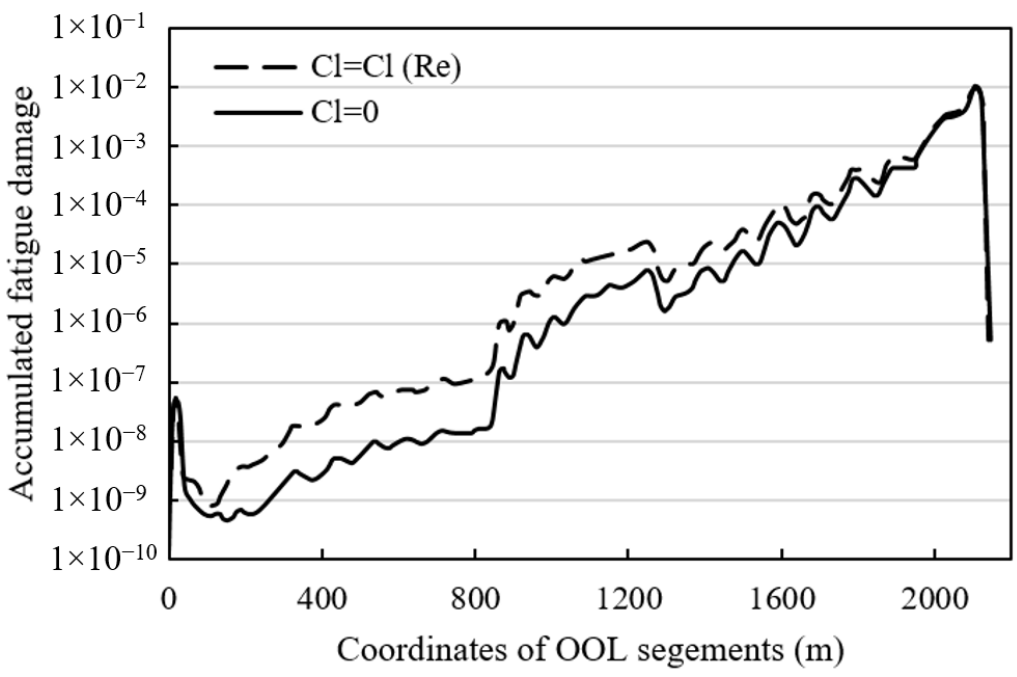

Figure 20. Fatigue damage distribution of OOL under current loads $\left(45^{\circ}\right)$.

As shown in Figure 20, under the action of current loads, the fatigue damage of the OOL mainly occurs near the CALM buoy. Although the fatigue damage curve has local maximum values near the FPSO and the segment covered by buoyancy material, it is far less than the fatigue damage near the CALM buoy. In addition, the vortex force caused by vortex-induced vibration has increased the fatigue damage in the middle part of the OOL by nearly 5-10 times, but it has little influence on the fatigue damage of the parts near the CALM buoy.

The fatigue damage distribution of the OOL when the current load direction is $90^{\circ}$ is shown in Figure 21. Comparing Figures 20 and 21, it can be found that the fatigue damage distribution along the OOL is different under different flow load directions, but the vortex-induced vibration can still increase the fatigue damage of the OOL by 5-10 times in the middle part. Meanwhile, the fatigue damage of the parts of the OOL near the CALM buoy is still much larger than other parts, and the effect of vortex-induced vibration is very small in this part. In addition, in the parts near the CALM buoy, the fatigue damage caused by $90^{\circ}$ current load is slightly less than that caused by $45^{\circ}$ current load.

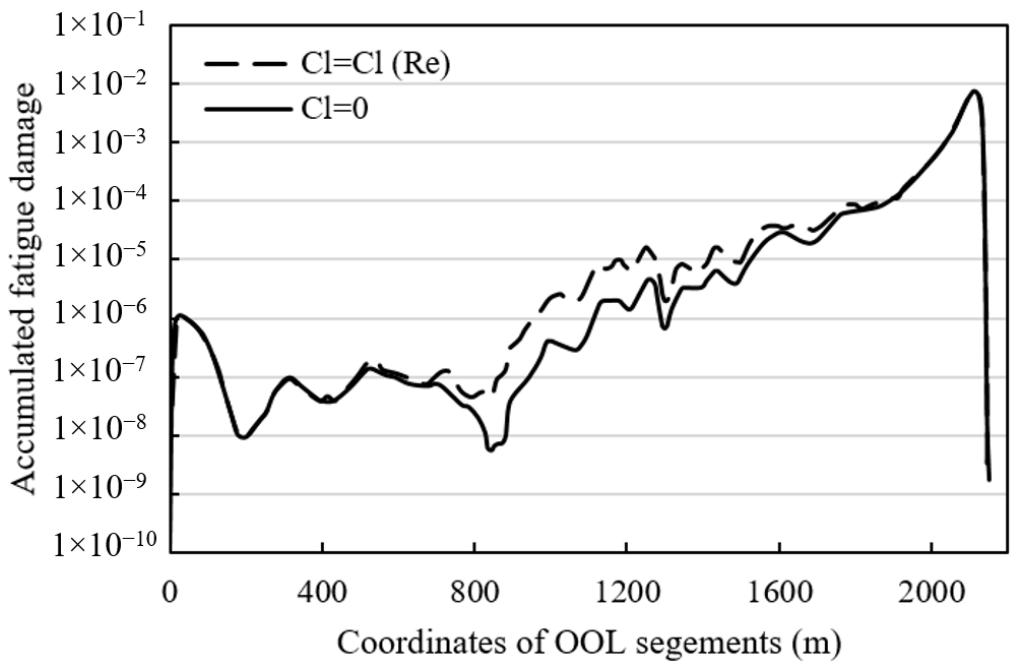

Figure 21. Fatigue damage distribution of OOL under current loads $\left(90^{\circ}\right)$.

According to the above analysis, it can be concluded that the fatigue damage caused by current load to the OOL is mainly concentrated in the part near the CALM buoy, and the flow load along the axial direction of the OOL can cause slightly larger fatigue damage. 
In addition, the effect of vortex-induced vibration is mainly concentrated in the middle part of the OOL, which can increase its fatigue damage by 5-10 times.

\section{Conclusions}

In this paper, fatigue analysis of oil offloading lines in the FPSO CALM buoy offloading system under wave and current loads in West Africa is carried out. The following conclusions can be drawn:

- The accumulated fatigue damage to the OOL is the largest near the area connecting the CALM buoy. Additionally, the section covered by buoyancy material has local maximum accumulated fatigue damage.

- Tension variation amplitude is a major factor for fatigue damage. Additionally, constrained boundary conditions may increase fatigue damage.

- $0^{\circ}$ waves cause greater fatigue damage. The fatigue damage of the OOL near the CALM buoy is mainly caused by the wind wave, and the fatigue damage of sections covered by buoyancy material is mainly caused by the swell.

- Appropriately reducing the cover length of buoyancy material and increasing the wall thickness can reduce fatigue damage. The outer diameter (with thickness unchanged) has little effect on the fatigue damage of the OOL.

- The effect of the shuttle tanker can increase the fatigue damage of the OOL near the CALM buoy by about 1.5 times, and the effect of vortex-induced vibration is mainly concentrated in the middle part of the OOL, which can increase fatigue damage by 5-10 times.

The present study reveals the influence of various factors on the oil offloading line's fatigue damage, such as motion and tension distribution, wave type, wave direction, coverage length of buoyancy material, outer diameter, wall thickness, shuttle tanker, and current loads, which can provide guidance for the fatigue design and optimization of pipeline layout and parameters in the FPSO oil offloading system. Moreover, the conclusions of this work can also serve as a reference for similar fatigue design in marine renewable energy technologies [28] or other offshore engineering cases, such as risers, mooring lines, and offshore cables.

Author Contributions: Conceptualization, W.N. and X.Z.; methodology, W.N.; software, X.Z.; validation, X.Z.; formal analysis, L.S.; investigation, W.N.; resources, L.S.; data curation, L.S.; writingoriginal draft preparation, W.N.; writing - review and editing, X.Z.; visualization, X.Z.; supervision, L.S.; project administration, L.S.; funding acquisition, W.N. All authors have read and agreed to the published version of the manuscript.

Funding: This research was funded by National Natural Science Foundation of China, Grant Number 52001112 .

Institutional Review Board Statement: Not applicable.

Informed Consent Statement: Not applicable.

Data Availability Statement: Not applicable.

Conflicts of Interest: The authors declare no conflict of interest.

\section{Nomenclature}

OOL Oil offloading line

FPSO Floating production storage and offloading

CALM Catenary anchor leg mooring

RAO Response amplification operator

$H_{S} \quad$ Significant wave height

$T_{p} \quad$ Wave period

L Length

D Diameter 
WT Wall thickness

$\mathrm{Cl} \quad$ Lift coefficient

Re Reynolds number

\section{References}

1. Ni, W.; Zhang, X.; Xu, F.; Zhang, W. Modified approximation method for structural failure probability analysis of high-dimensional systems. Ocean Eng. 2021, 237, 109486.1-109486.15. [CrossRef]

2. Zhang, X.; Sun, L.; Ma, C.; Fassezke, E.; Sun, H. A reliability evaluation method based on the weakest failure modes for side-by-side offloading mooring system of fpso. J. Loss Prev. Process Ind. 2016, 41, 129-143. [CrossRef]

3. Ni, W.; Zhang, X.; Xu, F.; Zhang, W.; Kang, Z. Numerical investigation of bifurcation characteristics under perturbations in vortex induced vibration of cylinder with two degrees of freedom. Ocean Eng. 2019, 188, 106318.1-106318.10. [CrossRef]

4. Liu, M.; Cross, C. Fatigue design and analysis of offshore pipelines and risers subjected to waves and currents. J. Offshore Mech. Arct. Eng. 2017, 140, 021701.1-021701.9.

5. Arzaghi, E.; Abbassi, R.; Garaniya, V.; Binns, J.; Chin, C.; Khakzad, N. Developing a dynamic model for pitting and corrosionfatigue damage of subsea pipelines. Ocean Eng. 2018, 150, 391-396. [CrossRef]

6. Zhang, Y.; Xiao, Z.; Luo, J. Fatigue crack growth investigation on offshore pipelines with three-dimensional interacting cracks. Geosci. Front. 2018, 9, 100-108. [CrossRef]

7. Al-Janabi, H.A.; Aubeny, C.P.; Chen, J.; Luo, M. Experimental measurement of monotonic and cyclic lateral resistance of risers and pipelines in gulf of mexico clays. Can. Geotech. J. 2020, 57, 1534-1549. [CrossRef]

8. Chaudhury, G. Double Catenary Offloading Lines for Deepwater Fields. In Proceedings of the Offshore Technology Conference, Houston, TX, USA, 6-9 May 2002.

9. Odru, P.; Poirette, Y.; Stassen, Y.; Marcoux, J.; Abergel, L. Composite Riser and Export Line Systems for Deep Offshore Applications In Proceedings of the Conference on Offshore Mechanics \& Arctic Engineering, Cancun, Mexico, 8-13 June 2003; pp. 147-156.

10. Montbarbon, S.; Quintin, S.H.; Deroux, G. Experience with new cost effective solutions to export oil from Deepwater floating production units using suspended pipelines. In Proceedings of the Offshore Technology Conference, Houston, TX, USA, 2-5 May 2005.

11. Drumond, G.P.; Pasqualino, I.P.; Pinheiro, B.C.; Estefen, S.F. Pipelines, risers and umbilicals failures: A literature review. Ocean Eng. 2018, 148, 412-425. [CrossRef]

12. He, Z.; Wei, Y.; Liu, S. Analysis of safe span length and fatigue life of submarine pipelines. China Ocean Eng. 2020, 34, 123-134. [CrossRef]

13. Fazeres-Ferradosa, T.; Rosa-Santos, P.; Taveira-Pinto, F.; Vanem, E.; Carvalho, H.; Correia, J. Editorial: Advanced research on offshore structures and foundation design: Part 1. Marit. Eng. 2019, 172, 118-123. [CrossRef]

14. Fazeres-Ferradosa, T.; Rosa-Santos, P.; Taveira-Pinto, F.; Pavlou, D.; Gao, F.; Carvalho, H.; Oliveira-Pinto, S. Preface: Advanced Research on Offshore Structures and Foundation Design: Part 2. Marit. Eng. 2020, 173, 96-99. [CrossRef]

15. Kang, Z.; Zhang, C.; Sun, L. Research on truncation method of fpso and offloading system in model test. Appl. Ocean Res. 2017, 67, 94-108. [CrossRef]

16. Kang, Z.; Zhang, C.; Ni, W.; Xu, X. Research on hydrodynamic calculation method of deepwater CALM buoy. In Proceedings of the International Offshore and Polar Engineering Conference, San Francisco, CA, USA, 25-30 June 2017; pp. $217-224$.

17. Amaechi, C.V.; Wang, F.; Hou, X.; Ye, J. Strength of submarine hoses in chinese-lantern configuration from hydrodynamic loads on calm buoy. Ocean Eng. 2018, 171, 429-442. [CrossRef]

18. Ni, W.; Zhang, X.; Zhang, W.; Liang, S. Time-domain numerical investigation of adaptive damping control for raft-type wave energy converters. Renew. Energy 2021, 175, 520-531. [CrossRef]

19. Oil Companies International Marine Forum. Prediction of Wind and Current Loads on VLCCs; Bermuda: London, UK, 1994; ISBN 13:9781856090421.

20. Fu, Y.F.; Zhang, J.Q.; Niu, D.T. Calculation Method for Flexural Damage of RC Beams Subjected to Cyclic Loads and Corrosive Actions. J. Highw. Transp. Res. Dev. Engl. Ed. 2019, 13, 44-51. [CrossRef]

21. Fouchereau, R.; Celeux, G.; Pamphile, P. Probabilistic modeling of S-N curves. Int. J. Fatigue 2014, 68, 217-223. [CrossRef]

22. DNV. Fatigue Strength Analysis of Offshore Steel Structures; DNV-RP-C203; Det Norske Veritas: Bærum, Norway, April 2010.

23. Aranuvachapun, S. Parameters of jonswap spectral model for surface gravity waves-II. Predictability from real data. Ocean Eng. 1987, 14, 101-115. [CrossRef]

24. Ochi, M.K.; Hubble, E.N. Six-parameter wave spectra. Coast. Eng. Proc. 1976, 1, 17. [CrossRef]

25. Guérin, P.; Le Buhan, P.; Heurtier, J. Investigations on Hydrodynamic and Mechanical Coupling Effects for Deepwater Offloading Buoy. In Proceedings of the Fourteenth International Offshore and Polar Engineering Conference, Toulon, France, 23-28 May 2004; pp. 374-379.

26. Kang, Z.; Ni, W.; Sun, L. A numerical investigation on capturing the maximum transverse amplitude in vortex induced vibration for low mass ratio. Mar. Struct. 2017, 52, 94-107. [CrossRef]

27. Norberg, C. Flow around a circular cylinder: Aspects of fluctuating lift. J. Fluids Struct. 2001, 15, 459-469. [CrossRef]

28. Taveira-Pinto, F.; Rosa-Santos, P.; Fazeres-Ferradosa, T. Marine renewable energy. Renew. Energy 2020, 150, 1160-1164. [CrossRef] 